\title{
Calcul des pertes par courants induits dans les conducteurs statoriques des machines asynchrones sans fer
}

\author{
A. Guéraud, B. Bandelier $\left(^{*}\right)$ et F. Rioux-Damidau \\ Laboratoire d'Electrotechnique des Universités Paris VI et XI, UA 0845, 91405 Orsay, France
}

(Reçu le 12 octobre 1988, révisé le 24 janvier 1989, accepté le 2 mars 1989)

\begin{abstract}
Résumé. - Les bobinages statoriques des machines sont généralement constitués de barres Roebell et plus rarement de barres torsadées. Le champ magnétique alternatif auquel ils sont soumis engendre des courants de Foucault locaux et, à l'intérieur des barres, des courants de circulation entre les différents conducteurs qui la constituent. On calcule ces courants dans le cas des machines sans fer en utilisant la modélisation des champs précédemment mise en place pour ces machines [1]. On montre ainsi que les pertes par courants de Foucault et par courants de circulation peuvent toujours être rendues négligeables par un choix approprié des conducteurs.

Abstract. - Statoric windings of electrical machines are generally made out of Roebell bars and, less frequently, of twisted conductor bars. The a.c. component of the magnetic field induces eddy currents within each elementary conductor and also induces currents which circulate between different conductors of the same bar. These currents are calculated, in the case of ironless asynchronous machines, using a computer simulation previously developed for such machines [1]. These calculations show that the eddy current losses, and the losses due to the induced circulating currents can always be made negligible with a good choice of the conductors.
\end{abstract}

\section{Introduction.}

Les machines asynchrones sans fer ont une structure très simple $[1,2]$. Elles comportent un rotor cylindrique massif en alliage d'aluminium entouré d'un bobinage statorique en cuivre. L'ensemble est logé dans une culasse magnétique qui assure la fermeture du flux. Le bobinage statorique est le siège de pertes Joule directes provoquées par le courant d'alimentation, que l'on calcule en supposant la densité de courant homogène. Mais les conducteurs du stator sont aussi le siège de pertes supplémentaires du fait de la présence de courants induits: nous nous proposons de les étudier, en distinguant deux sortes de pertes supplémentaires.

La première qui correspond aux pertes locales par courants de Foucault est due au fait qu'un conducteur massif plongé dans un champ alternatif est le

(*) Adresse actuelle : Laboratoire de Modèles de Physique Mathématique, Université de Tours, Parc de Grandmont, 37200 Tours, France. siège de «boucles» de courants induits. Dans les machines de structure classique, les lignes du champ sont concentrées dans les dents magnétiques, et celui-ci est petit dans les encoches où sont logés les conducteurs [3]. Ce phénomène est donc faible. Par contre, dans la machine que nous étudions, les conducteurs sont directement soumis au champ et les pertes par courants de Foucault qui y sont engendrées pourraient être importantes. Comme celles-ci sont proportionnelles au carré du diamètre des fils conducteurs du stator et qu'elles peuvent être assez facilement calculées en ordre de grandeur, on peut toujours les rendre très faibles en choisissant des conducteurs suffisamment fins.

La deuxième sorte de pertes que nous considérons est liée aux courants de circulation qui apparaissent dans les conducteurs lorsque le circuit formé par ces derniers définit une surface traversée par un flux. Comme les conducteurs sont en général torsadés, cela diminue le flux total traversant le circuit, mais il n'est pas possible d'évaluer l'ordre de grandeur du défaut à l'aide d'un calcul pas trop compliqué. Or lors de la conception d'une machine, il est indispen- 
sable de connaître les courants de circulation pour pouvoir procéder au choix des conducteurs : barres Roebell [4] qui ont une bonne rigidité et un bon coefficient de remplissage ou barres torsadées [5] qui satisfont un peu moins bien ces dernières qualités mais qui peuvent être fabriquées à partir de fils nettement plus fins. Les courants de Foucault dans ces dernières peuvent être plus faibles mais les courants de circulation, par construction, y sont beaucoup plus grands.

Cet article présente le calcul des courants de circulation et simultanément celui des courants de Foucault dans les conducteurs statoriques des machines sans fer. Les méthodes habituellement utilisées pour le calcul tridimensionnel de courants induits, à base d'éléments finis $[6,7,8]$, nécessiteraient dans un tel cas, vue la complexité de l'inducteur formé d'une multitude de fils, un calcul énorme. Nous utilisons ici une méthode de type analytique, à base de séries de Fourier. Elle permet, par des calculs très courts, de déterminer le champ magnétique en tout point du stator et d'en déduire les pertes supplémentaires associées, et cela quelle que soit la forme du courant d'alimentation [9].

\section{Pertes dans une barre.}

2.1 LeS barRes Roebell et torsadéEs. - Les barres Roebell présentent le mode le plus couramment utilisé pour réduire les courants de circulation [4]. Elles ont une section rectangulaire dont la plus grande dimension est orientée radialement dans la machine. Le motif de base est formé d'un fil qui aurait été enroulé avec un large pas sur une plaque extrêmement fine, la plaque ayant été enlevée après (cf. Fig. 1). La barre comprend plusieurs fils isolés, parallèles et jointifs, réalisant ainsi un ensemble compact.
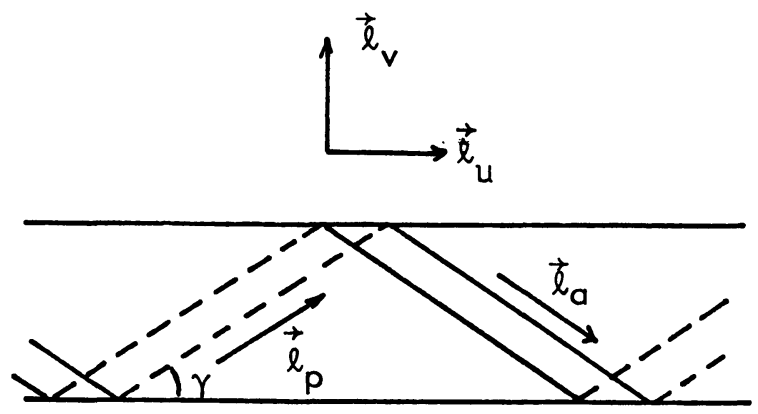

Fig. 1. - Motif de base des barres Roebell.

[Basic pattern of a Roebell bar.]

Les éléments constitutifs de base des barres torsadées sont des fils ronds isolés, dont le diamètre peut être très faible [5]. La géométrie "torsadée » est obtenue par la torsion d'un paquet de fils élémentaires, ce qui donne un toron. Une barre est elle-même formée de plusieurs torons auxquels on a fait également subir une torsion. L'ensemble est compacté sous forme de méplat.

Notons que le diamètre des fils élémentaires des barres torsadées peut être cinq fois plus faible que celui des éléments des barres Roebell, ce qui peut être très intéressant pour diminuer les "pertes Foucault ». Mais elles sont beaucoup moins aplaties et les courants de circulation, dus au flux qui traverse la surface séparant deux conducteurs élémentaires, y sont beaucoup plus importants.

\subsection{Pertes par courants de Foucault. - Nous} considérons un élément de barre plongé dans un champ alternatif $\mathcal{H}$, de pulsation $\omega$, supposé homogène sur cet élément; les fils constitutifs sont cylindriques, de rayon $r_{\mathrm{c}}$, de conductivité $\sigma$ et leur volume total est $d V$. Nous calculerons tout d'abord les pertes dans un fil de longueur $\mathrm{d} \boldsymbol{\ell}$ et de volume $\mathrm{d} v=\pi r_{\mathrm{c}}^{2} \mathrm{~d} \ell$ puis dans l'élément de barre Roebell ou torsadée.

2.2.1 Pertes dans un élément de fil. - La composante $\mathcal{H}_{\|}$de $\mathcal{H}$, parallèle à l'axe du fil, induit des courants $j_{\theta}(\rho, z)$ (cf. Figs. 2a et 2b) qui, d'après la loi de Faraday et la loi d'Ohm $(\mathbf{j}=\sigma \varepsilon)$, sont tels que :

$$
\begin{aligned}
& -\mu_{0} \frac{\partial \mathcal{H}_{\|}}{\partial t} \pi \rho^{2}=\int \varepsilon_{\theta} \mathrm{d} \ell=2 \pi \rho \varepsilon_{\theta}=2 \pi \rho \frac{j_{\theta}}{\sigma} \\
& \text { soit } \quad j_{\theta}(\rho, z)=-\frac{\mu_{0} \sigma \rho}{2} \frac{\partial \mathcal{H}_{\|}}{\partial t} .
\end{aligned}
$$

L'expression des courants $j_{z}(\rho, \theta)$ induits par $H_{\perp}$ s'obtient à l'aide d'un calcul analogue, en remarquant (cf. Fig. 2c) que le courant dans la portion $\mathrm{AB}$ de l'élément $\mathrm{d} \ell$ est annulé par le courant dans la portion $\mathrm{CD}$ de l'élément situé au-dessus :

$$
j_{z}(\rho, \theta)=-\mu_{0} \sigma \rho \sin \theta \frac{\partial \mathcal{H}_{\perp}}{\partial t} .
$$

En appliquant le théorème de superposition, nous pouvons écrire que les pertes engendrées par $j_{\theta}$ et $j_{z}$ dans l'élément de longueur $\mathrm{d} \ell$ ont une puissance égale à :

$$
\begin{aligned}
\mathrm{d} P_{\mathrm{F}}=\frac{\omega}{2 \pi} \frac{\mathrm{d} \ell}{\sigma} \int_{0}^{2 \pi / \omega} \mathrm{d} t \int_{0}^{r_{\mathrm{c}}} \int_{0}^{2 \pi}\left(\left|j_{\theta}(\rho, z)\right|^{2}+\right. \\
\left.+\left|j_{z}(\rho, \theta)\right|^{2}\right) \rho \mathrm{d} \rho \mathrm{d} \theta .
\end{aligned}
$$

Remplaçant $j_{\theta}$ et $j_{z}$ par (1) et (2) dans l'expression ci-dessus puis $H_{\perp}$ par son expression en fonction de $\mathfrak{H}$ et $\mathfrak{H}_{\|}$, ce qui allégera les calculs ultérieurs, on trouve [9] :

$$
\begin{aligned}
\mathrm{d} P_{\mathrm{F}}=\mathrm{d} v & \mu_{0}^{2} \sigma \frac{\omega}{16 \pi^{2}} \times \\
& \times \int_{0}^{2 \pi / \omega}\left(2\left|\frac{\mathrm{d} \mathscr{H}}{\mathrm{d} t}\right|^{2}-\left|\frac{\mathrm{d} \mathcal{H}_{\|}}{\mathrm{d} t}\right|^{2}\right) \mathrm{d} t .
\end{aligned}
$$




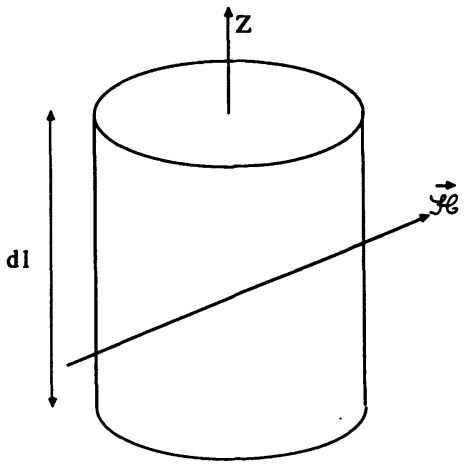

(a)

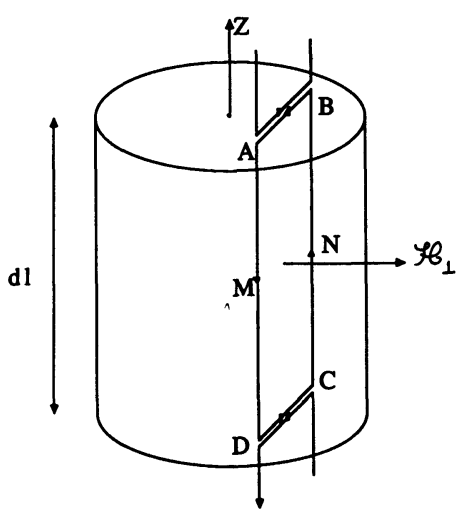

(c)

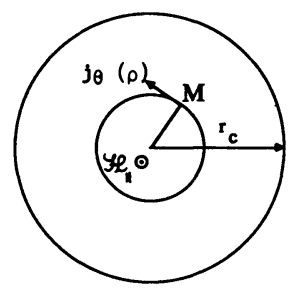

(b)

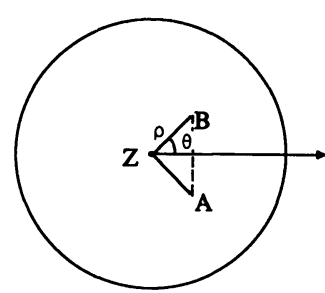

(d)
Fig. 2. - Courants induits par le champ magnétique $\mathfrak{H}$.

[Currents induced by the magnetic field $\mathfrak{H}$.]

2.2.2 Pertes dans un élément de barre Roebell. - Le fil constitutif à l'intérieur de la barre est défini soit par le vecteur unitaire $\mathbf{e}_{\mathrm{p}}$, soit par $\mathbf{e}_{\mathrm{a}}$ (Fig. 1), avec, si nous appelons $\gamma$ l'angle d'inclinaison du fil par rapport à la barre :

$$
\mathbf{e}_{\mathrm{p}, \mathrm{a}}=\cos \gamma \mathbf{e}_{u} \pm \sin \gamma \mathbf{e}_{v}
$$

(+ pour $\mathbf{e}_{\mathrm{p}},-$ pour $\mathbf{e}_{\mathrm{a}}$ ), le trièdre $\mathbf{e}_{u}, \mathbf{e}_{v}, \mathbf{e}_{w}$ étant un repère lié à la barre.

La formule générale des pertes s'applique pour chacune des deux faces. En remarquant que :

$$
\begin{array}{r}
\left|\frac{\mathrm{d}}{\mathrm{d} t}\left(\mathscr{H}_{\|}\right)_{\mathrm{p}, \mathrm{a}}\right|^{2}=\cos ^{2} \gamma\left|\frac{\mathrm{d} \mathscr{H}_{u}}{\mathrm{~d} t}\right|^{2}+\sin ^{2} \gamma\left|\frac{\mathrm{d} \mathscr{H}_{v}}{\mathrm{~d} t}\right|^{2} \pm \\
\pm 2 \sin \gamma \cos \gamma \frac{\mathrm{d}_{u}}{\mathrm{~d} t} \frac{\mathrm{d}^{2} \mathscr{H}_{v}}{\mathrm{~d} t}
\end{array}
$$

nous en déduisons les pertes par courants de Foucault dans l'élément de volume $\mathrm{d} V$ de la barre Roebell :

$$
\begin{aligned}
\mathrm{d} P_{\mathrm{F}} & =\mathrm{d} V \mu_{0}^{2} \sigma r_{\mathrm{c}}^{2} \frac{\omega}{16 \pi} \int_{0}^{2 \pi / \omega}\left(4\left|\frac{\mathrm{d} \mathscr{H}}{\mathrm{d} t}\right|^{2}-\right. \\
& \left.-2\left(\cos ^{2} \gamma\left|\frac{\mathrm{d} \mathscr{H}_{u}}{\mathrm{~d} t}\right|^{2}+\sin ^{2} \gamma\left|\frac{\mathrm{d} \mathscr{H}_{v}}{\mathrm{~d} t}\right|^{2}\right)\right) \mathrm{d} t
\end{aligned}
$$

le champ étant pris au centre de l'élément $\mathrm{d} V$.
2.2.3 Pertes dans un élément de barre torsadée. Les barres torsadées étant formées d'un assemblage de torons, nous rechercherons tout d'abord les courants de Foucault dans un élément de toron et, plus précisément, pour les fils situés sur la couche

Fil b

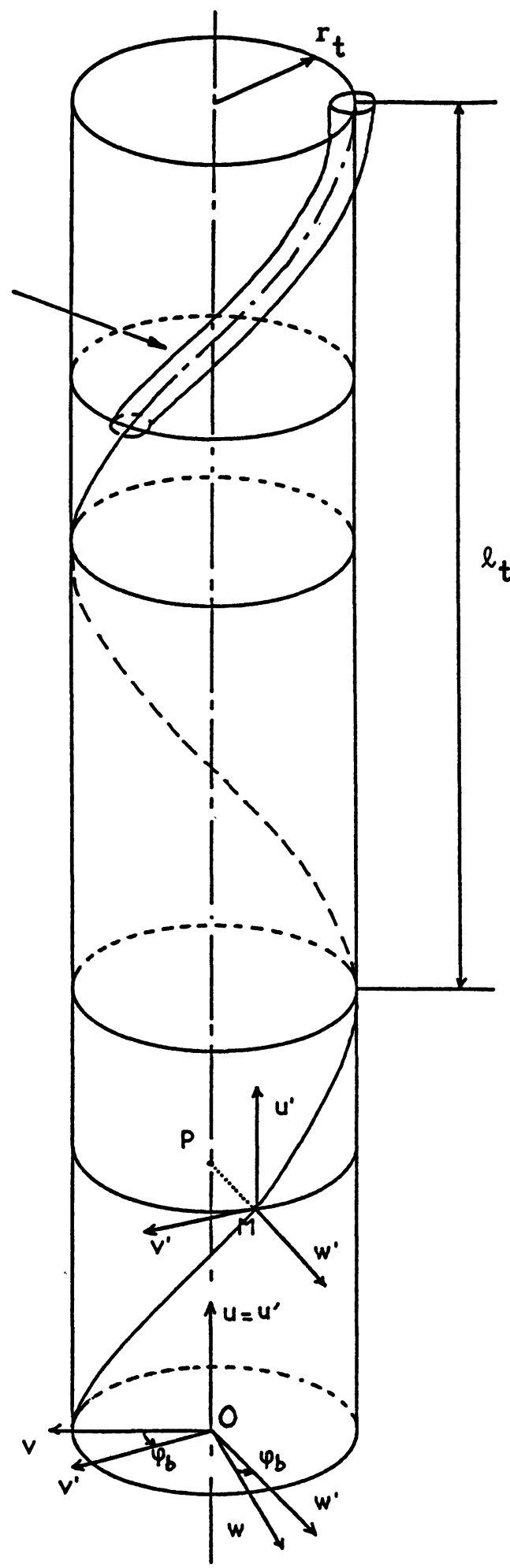

Fig. 3. - Un toron d'une barre torsadée.

[A twine of a twisted bar.] 
externe du toron, au rayon $r_{t}$ (cf. Fig. 3). En se plaçant dans le référentiel lié au toron, nous avons :

$$
\mathrm{d} \boldsymbol{\ell}=\left(a \mathbf{e}_{u}+b \mathbf{e}_{v}+c \mathbf{e}_{w}\right) \mathrm{d} \boldsymbol{\ell}
$$

où

$$
\begin{aligned}
a & =\sqrt{\frac{\ell_{\mathrm{t}}^{2}}{4 \pi^{2} r_{\mathrm{c}}^{2}+\ell_{\mathrm{t}}^{2}}} / m \\
b & =\sin \left(2 \pi u / \ell_{\mathrm{t}}\right) / m \\
c & =\cos \left(2 \pi u / \ell_{\mathrm{t}}\right) / m \\
m^{2} & =a^{2}+b^{2}+c^{2} .
\end{aligned}
$$

Les fils externes étant les plus nombreux et les courants de Foucault dans les fils internes différant peu de ceux existant dans les fils externes (seule la valeur de $r_{\mathrm{t}}$ change), nous admettrons qu'ils y sont égaux. Les différents éléments de torons de l'élément de barre $\mathrm{d} V$ étant soumis au même champ, on obtient ainsi, à partir de (3) :

$$
\begin{aligned}
& \mathrm{d} P_{\mathrm{F}}=\mathrm{d} V \mu_{0}^{2} \sigma r_{\mathrm{c}}^{2} \frac{\omega}{16 \pi} \int_{0}^{2 \pi / \omega}\left(2\left|\frac{\mathrm{d} \mathscr{H}}{\mathrm{d} t}\right|^{2}-\right. \\
& \left.-a^{2}\left|\frac{\mathrm{d} \mathscr{X}_{u}}{\mathrm{~d} t}\right|^{2}-b^{2}\left|\frac{\mathrm{d} \mathscr{X}_{v}}{\mathrm{~d} t}\right|^{2}-c^{2}\left|\frac{\mathrm{d} \mathscr{X}_{w}}{\mathrm{~d} t}\right|^{2}\right) \mathrm{d} t .
\end{aligned}
$$

\subsection{PERTES PAR COURANTS DE CIRCULATION. -} Une barre torsadée est constituée d'un ensemble de petits torons formant eux-mêmes un gros toron aplati par la construction. Pour faciliter son étude, nous négligerons cette déformation et considérerons que nous avons simplement affaire à un gros toron, comme celui représenté figure $3,2 r_{\mathrm{t}}$ correspondant à la plus grande dimension de la barre, puis nous calculerons les courants dans les fils comme s'ils étaient tous sur la couche externe du toron. L'ensemble de ces hypothèses fournit une majoration des pertes.

Une barre Roebell ayant un motif de base encore plus aplati est soumise à des courants beaucoup plus faibles; nous n'examinerons cette configuration qu'après avoir déterminé les ordres de grandeur des courants dans les barres torsadées (cf. paragraphe 3 ).

Nous déterminerons donc tout d'abord l'expression du flux magnétique $\Phi_{b}$ à travers la surface $S$ délimitée par l'axe du toron et l'axe du fil $b$ (Fig. 3) et nous en déduirons les courants que les divers flux font circuler dans les fils reliés en parallèle.

Comme on peut le constater sur la figure 4, la surface élémentaire $d S$ n'est pas plane. Aussi, nous la décomposerons en trois surfaces élémentaires planes dans le référentiel $M \boldsymbol{u}^{\prime} \boldsymbol{v}^{\prime} \boldsymbol{w}^{\prime}$ lié au fil $\left(\mathrm{M} u^{\prime} / / \mathrm{O} u\right)$. Soit $\varphi_{b}$ l'angle $\left(\mathrm{O} v, \mathrm{M}^{\prime}\right)$, égal à $\left(\mathrm{O} w, \mathrm{Mw}^{\prime}\right)$; il vaut :

avec

$$
\begin{aligned}
\varphi_{b} & =\varphi_{0 b}+2 \pi u / \ell_{\mathrm{t}} \\
u & =z / \cos \alpha
\end{aligned}
$$
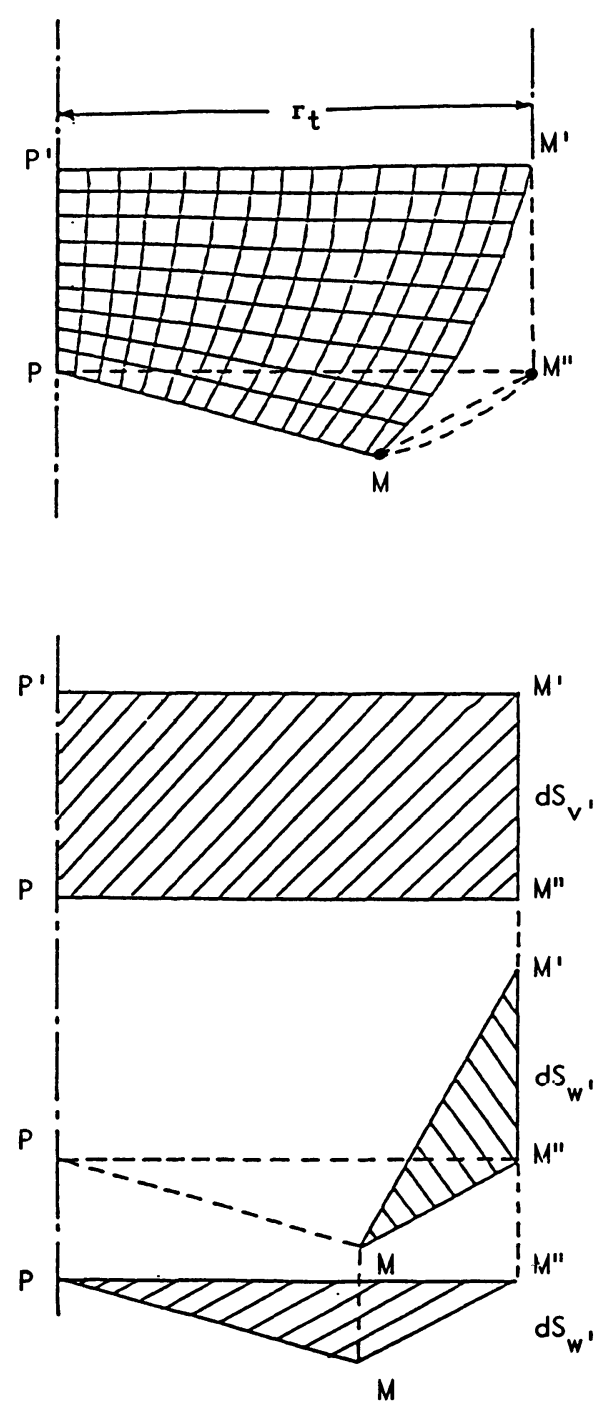

Fig. 4. - La surface de passage du flux élémentaire $\mathrm{d} \Phi_{b}$.

[The area crossed by the elementary flux $\mathrm{d} \Phi_{b}$.]

$\varphi_{0 b}$ étant l'angle pour $u=z=0$, et $\alpha$ l'angle du toron par rapport à l'axe $\mathrm{O} z$ de la machine. Alors :

$$
\begin{aligned}
\mathrm{d} S_{u^{\prime}} & =r_{\mathrm{t}}^{2} \mathrm{~d} \varphi_{b} / 2=\pi r_{\mathrm{t}}^{2} \mathrm{~d} u / \ell_{\mathrm{t}} \\
\mathrm{d} S_{v^{\prime}} & =r_{\mathrm{t}} \mathrm{d} u \\
\mathrm{~d} S_{w^{\prime}} & =r_{\mathrm{t}} \mathrm{d} \varphi_{b} \mathrm{~d} u=2 \pi r_{\mathrm{t}} / \ell_{\mathrm{t}}(\mathrm{d} u)^{2} .
\end{aligned}
$$

L'aire $\mathrm{d} S_{w^{\prime}}$, qui est du second ordre en $\mathrm{d} u$, est négligeable par rapport à $\mathrm{d} S_{u^{\prime}}$ et $\mathrm{d} S_{v^{\prime}}$. Par suite

$$
\begin{aligned}
\mathrm{d} \Phi_{b}=\mu_{0}\left[\mathscr{H}_{u} \pi r_{\mathrm{t}} / \ell_{\mathrm{t}}\right. & +H_{v} \cos \varphi_{b}+ \\
& \left.+\mathcal{H}_{w} \sin \varphi_{b}\right] r_{\mathrm{t}} \mathrm{d} z / \cos \alpha .
\end{aligned}
$$

En supposant, comme en 2.2 , le champ $\mathscr{H}$ constant sur tout un élément de toron, les divers flux élémentaires $\mathrm{d} \Phi_{b}$ embrassés par les divers fils $b$ du toron ne différeront que par la valeur de l'angle $\varphi_{b}$, c'est-àdire par celle de $\varphi_{0 b}$ qui, d'un fil à l'autre, varie de 
$2 \pi / B$ où $B$ est le nombre de fils de la couche à la distance $r_{\mathrm{t}}$ :

$$
\begin{aligned}
\varphi_{0 b} & =\varphi_{01}+(b-1) 2 \pi / B \\
\varphi_{b} & =\varphi_{01}+(b-1) 2 \pi / B+2 \pi z / \ell_{\mathrm{t}} \cos \alpha \\
& =\varphi_{1}+(b-1) 2 \pi / B
\end{aligned}
$$

On peut ainsi calculer pour chaque fil :

$$
\Phi_{b}=\int \mathrm{d} \Phi_{b}
$$

l'intégrale portant sur la longueur de la machine, la valeur de Je étant prise sur l'axe du toron.

Les différents fils sont reliés en parallèle à leurs extrémités et la tension $U$ à leurs bornes est donc indépendante du fil considéré. Elle est liée à $\Phi_{b}$ et à la densité de courant $j_{b}$ par :

$$
U=-\frac{\partial \Phi_{b}}{\partial t}+\frac{\ell_{0}}{\sigma} j_{b}
$$

$\ell_{0}$ étant la longueur du fil.

Par raison de symétrie, ces densités de courant diffèrent, d'un fil à l'autre, d'une phase $2 \pi / B$; on peut donc les écrire sous forme :

$$
j_{b}=j^{\prime} \cos \varphi_{b}+j^{\prime \prime} \sin \varphi_{b} .
$$

Ceci assure que la somme des courants dans les $B$ fils est nulle ; ce sont bien des courants de circulation interne au toron, ne sortant pas vers l'extérieur.

Reportant (8) et (10) dans (11) et remarquant que l'équation obtenue doit être vraie quel que soit $b$, on en déduit :

$$
\begin{gathered}
U=\frac{\mu_{0} \pi r_{\mathrm{t}}^{2}}{\ell_{\mathrm{t}} \cos \alpha} \int \frac{\mathrm{d} \mathscr{H}_{u}}{\mathrm{~d} t} \mathrm{~d} z \\
j_{b}=\frac{\sigma}{l_{0}} \frac{\mu_{0} r_{\mathrm{t}}}{\cos \alpha} \frac{\mathrm{d}}{\mathrm{d} t} \int\left(\mathscr{H}_{v} \cos \varphi_{b}+\mathscr{H}_{w} \sin \varphi_{b}\right) \mathrm{d} z
\end{gathered}
$$

les intégrales portant sur la longueur de la machine.

\section{Méthode de calcul des pertes dans les machines sans fer.}

Nous reporterons dans les expressions que nous venons d'établir les valeurs des champs magnétiques que nous avons calculées précédemment [1] et en déduirons les pertes.

3.1 ChAMP DANS LA MACHINE SANS FER. - La modélisation décrite en [1] et que nous rappelons brièvement considère le système représenté figure 5 , qui est constitué d'une infinité de machines, réguliè-

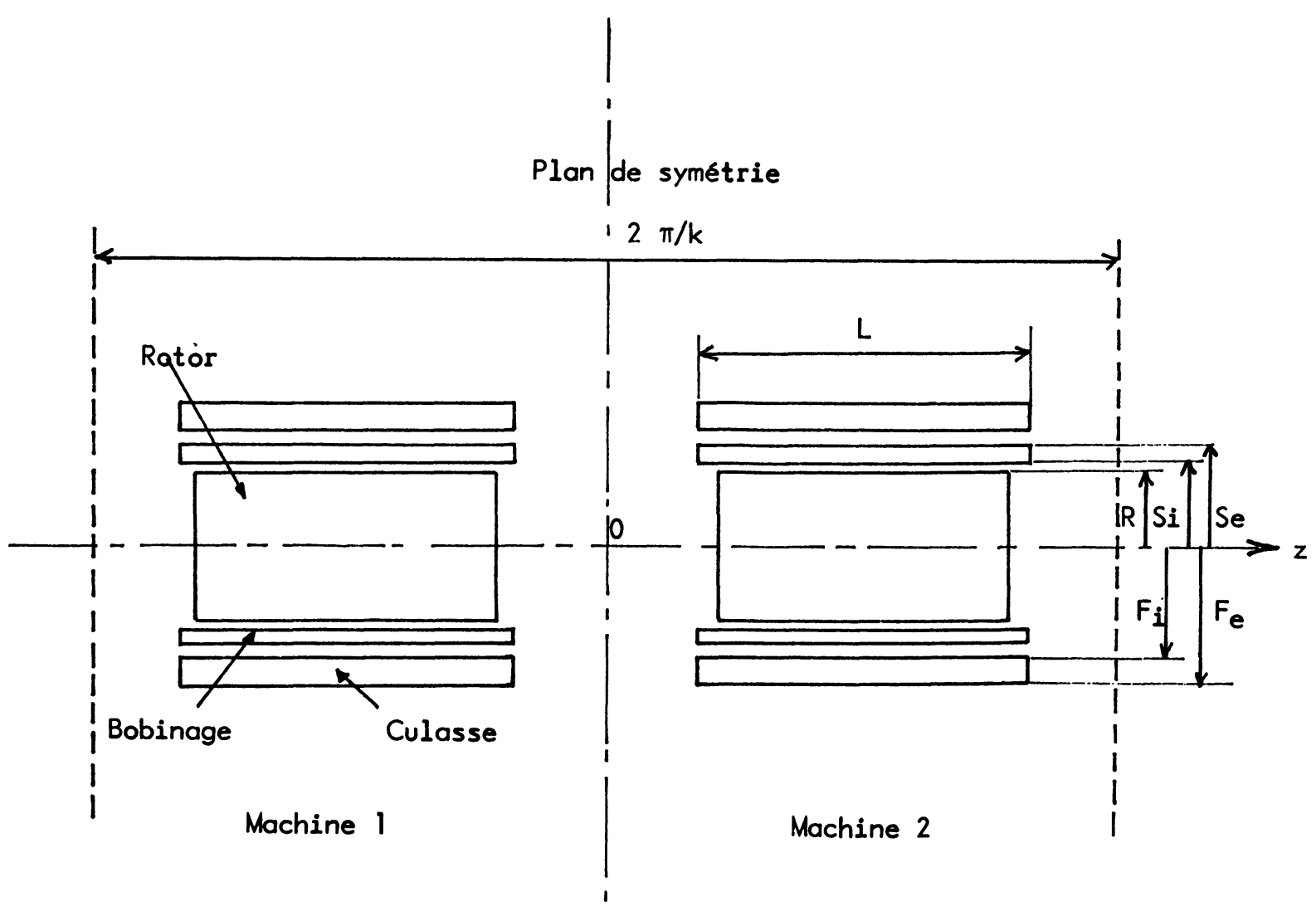

Fig. 5. - Le système adopté pour modéliser la machine.

[The modeled system.] 
rement alignées le long de l'axe $\mathrm{O} z$ et suffisamment éloignées l'une de l'autre pour ne pas interagir. Deux machines consécutives sont symétriques par rapport à un plan perpendiculaire à $\mathrm{Oz}$. Toutes les grandeurs du système et en particulier le champ $J$ sont alors décomposées en série de Fourier de $\theta, z$, $t$ :

$$
\mathcal{H}(r, \theta, z, t)=\sum_{n \ell q \in \mathbf{Z}^{3}} \mathbf{H}_{n \ell q}(\rho) \mathrm{e}^{i(n \omega t+\ell \theta+q k z)}
$$

$I(\omega t)$

$-2 \pi / k$ est le pas suivant $\mathrm{O} z$ du système modélisé. Il comprend deux machines.

La résolution des équations de Maxwell détermine les coefficients de Fourier $\mathbf{H}_{n \ell q}$. Dans cette description, le bobinage est décrit à partir du courant $\mathbf{J}$ existant dans la première bobine de la première paire de pôles $\mathrm{J}(\theta, \rho, z, t)=I(\omega t) \mathbf{e}_{u}(\theta, \rho, u) ;$ le courant dans les autres bobines est obtenu en tenant compte d'une part du décalage spatial de ces autres bobines et d'autre part du déphasage temporel des courants d'alimentation équilibrés. Le vecteur unitaire $\mathbf{e}_{u}$, associé aux barres Roebell ou torsadées, est décomposé en série de Fourier spatiale de $z$ et $\theta$, et $I$ en série de Fourier temporelle :

$$
I(\omega t)=\sum_{n \in \mathbf{Z}} I_{n} \mathrm{e}^{i n \omega t}
$$

avec

$$
I_{n}=I_{-n}^{*} \text {. }
$$

Comme les machines ont un comportement linéaire vis-à-vis du courant, on montre que :

$$
\mathbf{H}_{n \ell q}=\mathbf{h}_{\ell_{q}}(n) I_{n} \text {. }
$$

Les symétries existant dans le système entraînent [10] :

$$
\begin{aligned}
& \mathbf{h}_{\ell, q}(n)=\mathbf{h}_{-\ell,-q}^{*}(-n)=\mathbf{h}_{-\ell, q}^{*}(n)=\mathbf{h}_{\ell,-q}(n) \\
& \text { avec } \quad \ell=r p \quad(r \in \mathbb{Z}),
\end{aligned}
$$

où $p$ est le nombre de paires de pôles, et

$$
\frac{\ell}{p}+n=m f \quad(f \in \mathbb{Z})
$$

où $m$ est le nombre de phases.

3.2 CALCUl DES PERTES PAR COURANTS DE FOUCAULT. - Il nous faut intégrer les expressions (5) ou (7) sur le volume du bobinage, en tenant compte du coefficient de remplissage $K_{\mathrm{r}}$ du stator.

Il apparaît tout d'abord l'intégrale :

$$
\int_{S_{\mathrm{i}}}^{S_{\mathrm{e}}} \mathrm{d} \rho \int_{0}^{2 \pi} \mathrm{d} \theta \int_{L} \mathrm{~d} z \int_{0}^{2 \pi / \omega} \mathrm{d} t\left|\frac{\mathrm{d} \mathscr{H}}{\mathrm{d} t}\right|^{2}
$$

$\int_{L}$ signifiant que l'on intègre sur les deux longueurs de machine comprises dans un pas du système puis que l'on divise le résultat par 2. Reportant la décomposition (15) dans cette intégrale, on obtient :

$$
\begin{aligned}
& 8 \pi^{2} n^{2} \omega^{2}\left|I_{n}\right|^{2} \int_{S_{\mathrm{i}}}^{S_{\mathrm{e}}} \mathrm{d} \rho \sum_{n \ell_{q}} \mathbf{h}_{\ell q}(n) \cdot \mathbf{h}_{-\ell-q}(-n)+ \\
& \quad+8 \pi^{2} n^{2} \omega^{2}\left|I_{n}\right|^{2} \int_{S_{\mathrm{i}}}^{S_{\mathrm{e}}} \mathrm{d} \rho \\
& \quad \times \sum_{n q \ell_{q^{\prime} \neq q}}(-1)^{\left(q+q^{\prime}\right) / 2} \frac{\sin \left(\left(q+q^{\prime}\right) k L / 2\right)}{\left(q+q^{\prime}\right) k} \\
& \quad \times \mathbf{h}_{\ell_{q}}(n) \cdot \mathbf{h}_{-\ell_{q}}(-n) .
\end{aligned}
$$

Le premier terme est le même que si l'intégrale en $z$, $\int_{L}$ avait porté sur un pas complet du système modélisé et non sur la seule longueur de la machine.

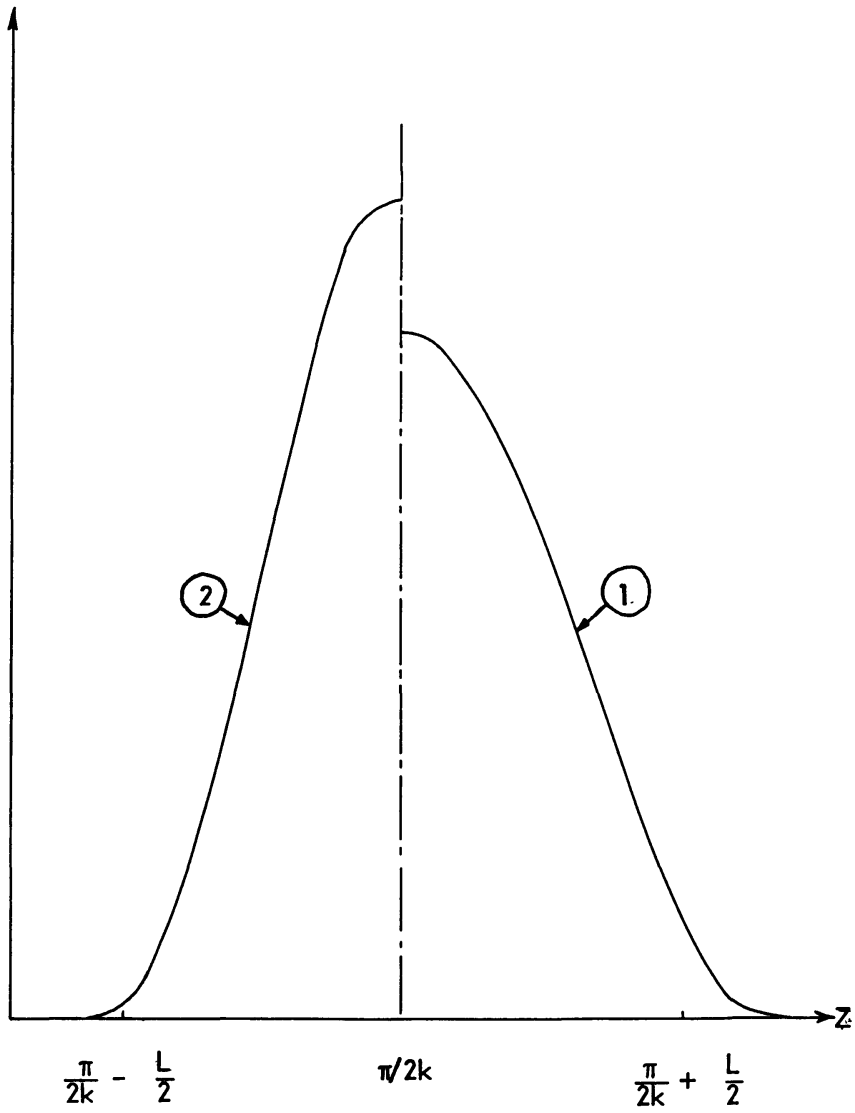

Fig. 6. - Valeur du champ (1) et du carré du champ (2) pour $\rho=S_{\mathrm{i}}$ (rayon interne du bobinage de la machine) le long d'une génératrice. La machine est située entre les points $\frac{\pi}{2 k}-\frac{L}{2}$ et $\frac{\pi}{2 k}+\frac{L}{2}$.

[Values of the field (1) and of its square (2) for $\rho=S_{\mathrm{i}}$ (internal radius of the stator) along a generatrix. The machine is placed between $\frac{\pi}{2 k}-\frac{L}{2}$ and $\frac{\pi}{2 k}+$ $\left.\frac{L}{2}.\right]$ 
Le second terme est lié à la présence d'un champ à l'extérieur du bobinage entre les rayons $S_{\mathrm{i}}$ et $S_{\mathrm{e}}$. La figure 6 montre ce champ dans le prototype pour $\rho=S_{\mathrm{i}}$ (rayon pour lequel il est le plus grand) lorsqu'on se déplace parallèlement à $\mathrm{O} z$; on voit que $|\mathcal{H C}|^{2}$ est quasiment nul à l'extérieur du bobinage et que ce peut donc être une excellente approximation de négliger le deuxième terme de (21).

Pour l'intégrale avec $\mathfrak{H}_{u}$ au lieu de $\mathfrak{H}$, nous obtenons des expressions similaires avec $\left(h_{u}\right)_{\ell_{q}}$ au lieu de $\mathbf{h}_{\ell q}$. Or:

$$
\left(h_{u}\right)_{\ell q}=\sum_{\ell^{\prime} q^{\prime}} \mathbf{h}_{\ell^{\prime} q^{\prime}} \cdot\left(\mathbf{e}_{u}\right)_{\ell-\ell^{\prime}, q-q^{\prime}} .
$$

Le calcul de cette expression est lourd car il nécessite de poursuivre la sommation sur $\ell^{\prime}$ très loin, de telle sorte que l'on tienne compte des termes avec $\left|\ell^{\prime}-\ell\right| \gg \ell$ ce qui entraîne $\left|\ell^{\prime}\right| \gg|2 \ell|$; il en est de même pour $q^{\prime}$. Le même problème se retrouve avec $\mathcal{H}_{v}$ et $\mathfrak{H}_{w}$. Or, dans le prototype, le bobinage est constitué de deux nappes de conducteurs distinctes superposées, en forme d'hélice à pas diamétral (cf. Fig. 7). Aussi cette forme simple rend plus facile l'évaluation directe de $\mathfrak{H}_{u}, \mathfrak{H}_{v}$ et $\mathfrak{H}_{w}$ à partir de $\mathfrak{H}$. On a :

$$
\begin{aligned}
\mathscr{H}_{u} & =\varepsilon \mathscr{H}_{z} \cos \alpha+\mathscr{H}_{\theta} \sin \alpha \\
\mathscr{H}_{v} & =\mathscr{H}_{\rho} \\
\mathscr{H}_{w} & =-\mathscr{H}_{z} \sin \alpha+\varepsilon \mathscr{H}_{\theta} \cos \alpha
\end{aligned}
$$

avec $\varepsilon=1$ pour la première nappe et $\varepsilon=-1$ pour la seconde nappe. Comme la modélisation des machines donne directement $\left(h_{\rho}\right)_{\ell_{q}},\left(h_{\theta}\right)_{\ell_{q}},\left(h_{z}\right)_{\ell_{q}}$, nous traitons les intégrales qui se présentent de la même manière que l'intégrale (20) en négligeant donc l'équivalent du second terme de (21).

En conclusion, le calcul des pertes Foucault se fait en intégrant les expressions (5) ou (7) sur toute la longueur de la machine et pour le bobinage hélicoïdal $\mathfrak{H}_{u}, \mathfrak{H}_{v}$ et $\mathfrak{H}_{w}$ en fonction des composantes de $\mathfrak{H}_{\text {. }}$

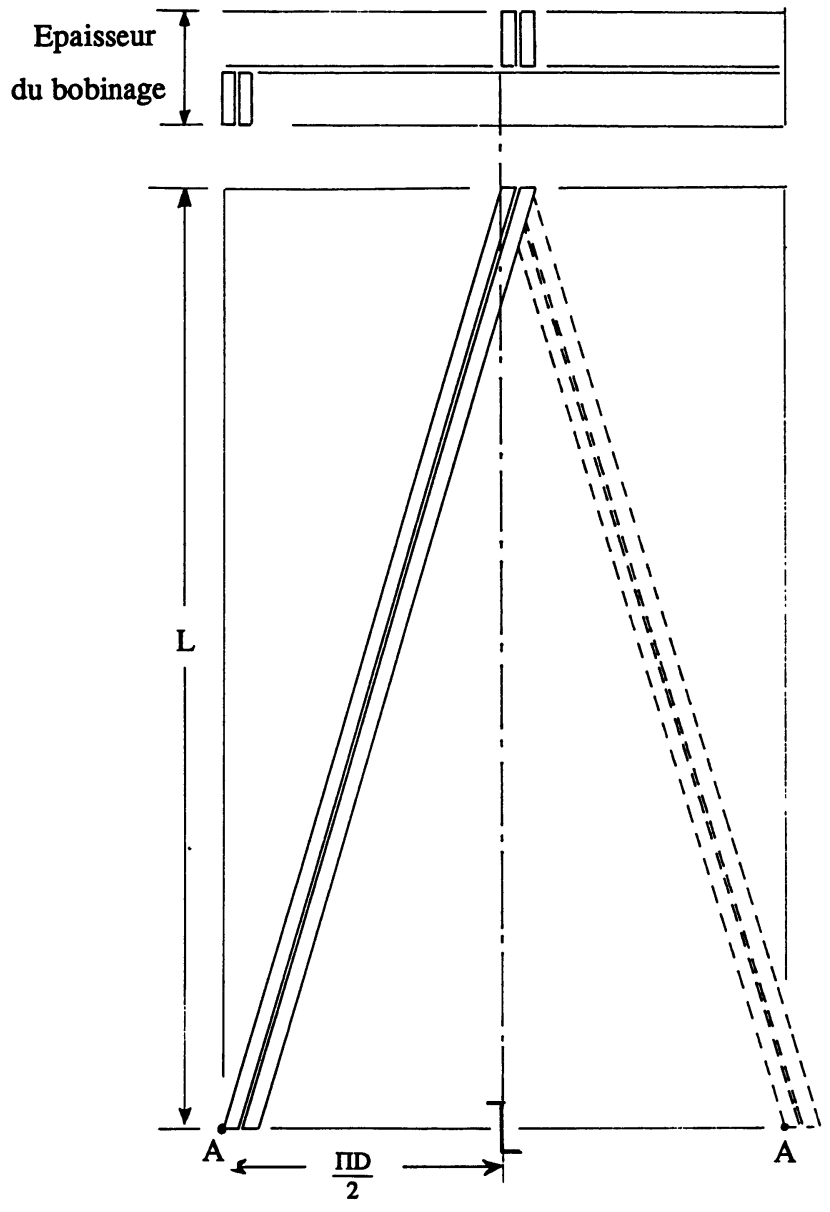

Fig. 7. - Vue développée et vue en coupe de deux conducteurs des deux nappes superposées constituant le bobinage.

[Developed view and cross-section of two conductors of the two superposed sheets of the winding.]

3.3 Calcul des Courants de CiRCulation. Nous calculerons tout d'abord le courant donné par l'expression (14).

En considérant le bobinage hélicoïdal pour lequel $\mathfrak{J}_{u}$ et $\varkappa_{w}$ sont donnés par (23), et en remplaçant les composantes de He par leur développement (15) en série de Fourier, (14) devient :

$$
\begin{aligned}
j_{b}=\mu_{0} \sigma \frac{R}{\ell_{0} \cos \alpha} \sum_{n \ell q \in \mathbf{Z}^{3}} i n \omega \mathrm{e}^{i n \omega t} \times \int & \mathrm{d} z \mathrm{e}^{i(\ell \theta+q k z)} \times \\
& \times I_{n}\left(h_{\rho, \ell_{q}} \cos \varphi_{b}+\left(-h_{z} \sin \alpha+\varepsilon h_{\theta} \cos \alpha\right) \ell_{q} \sin \varphi_{b}\right) \mathrm{d} z
\end{aligned}
$$

où $\varphi_{b}$ dépend de $z$ (il est donné par (9)), $\theta$ et $z$ sont les coordonnées du point $P$ situé sur l'axe du toron (cf. Fig. 3) avec $\theta=\theta_{0}+\varepsilon \pi z / L, \theta_{0}$ dépendant du toron considéré.

En posant :

$$
\nu_{ \pm} k=\varepsilon \ell \frac{\pi}{L}+q k \pm \frac{2 \pi}{\ell_{0} \cos \alpha}
$$


et en calculant le courant dans la machine située entre $z=\frac{\pi}{2 k}-\frac{L}{2}$ et $z=\frac{\pi}{2 k}+\frac{L}{2}$, on obtient :

$$
\begin{array}{r}
j_{b}=-\frac{\mu_{0} \sigma R}{\ell_{0} \cos \alpha} \sum_{n \ell_{q}} i n \omega \mathrm{e}^{i n \omega t} I_{n}\left[\frac{\sin \nu_{+} k L / 2}{\nu_{+} k} \mathrm{e}^{i \nu_{+} \frac{\pi}{2}}\left(h_{\rho}+i h_{z} \sin \alpha-i \varepsilon h_{\theta} \cos \alpha\right) \ell_{q} \mathrm{e}^{i \varphi_{0 b}}+\right. \\
\left.+\frac{\sin \nu_{-} k L / 2}{\nu_{-} k} \mathrm{e}^{i \nu_{-} \frac{\pi}{2}}\left(h_{\rho}-i h_{z} \sin \alpha+i \varepsilon h_{\theta} \cos \alpha\right) \ell_{q} \mathrm{e}^{-i \varphi_{0 b}}\right] \\
=\sum_{n}\left(j_{n+}^{\prime}+i j_{n+}^{\prime \prime}\right) \mathrm{e}^{i \varphi_{0 b}}+\left(j_{n-}^{\prime}+i j_{n-}^{\prime \prime}\right) \mathrm{e}^{-i \varphi_{0 b}}
\end{array}
$$

Il faut remarquer que $j_{n+}^{\prime}, j_{n+}^{\prime \prime}, j_{n-}^{\prime}, j_{n-}^{\prime \prime}$ sont les mêmes pour tous les fils d'un même toron.

Vues les propriétés de symétrie (18) des coefficients de Fourier de $\mathscr{H}$, on peut constater que cette expression est bien réelle et qu'elle peut se mettre sous la forme :

avec

$$
\begin{aligned}
j_{b} & =\sum_{n} j_{b n} \mathrm{e}^{i n \omega t} \\
j_{b n} & =j_{b-n}^{*} .
\end{aligned}
$$

La moyenne quadratique du courant est alors :

$$
\begin{aligned}
\overline{j_{b}^{2}}= & \sum_{n}\left|j_{b n}\right|^{2} \\
= & \sum_{n}\left(j_{n+}^{\prime 2}+j_{n+}^{\prime \prime^{2}}+j_{n-}^{\prime 2}+j_{n-}^{\prime \prime \prime}\right) \\
& +2 \cos \left(2 \varphi_{0 b}\right)\left(j_{n+}^{\prime} j_{n-}^{\prime}+j_{n+}^{\prime \prime} j_{n-}^{\prime \prime}\right) \\
& +2 \sin \left(2 \varphi_{0 b}\right)\left(j_{n+}^{\prime} j_{n-}^{\prime \prime}-j_{n-}^{\prime} j_{n+}^{\prime \prime}\right) .
\end{aligned}
$$

Cette expression comporte un terme indépendant du fil considéré et deux termes en $\varphi_{0 b}$, qui dépendent à la fois du fil considéré et de $\varphi_{01}$, c'est-à-dire de la position du premier fil dans le toron. En fait, les termes fluctuants se compenseront sur l'ensemble des fils et l'on pourra calculer les pertes en ne retenant que le premier terme de (27).

\section{Valeur des pertes dans les machines sans fer.}

4.1 PeRtes DANS LE PROTOTYPE. - Il existe au Laboratoire d'Electrotechnique d'Orsay un prototype de machine cylindrique asynchrone sans fer qui délivre $200 \mathrm{~kW}$ à $30000 \mathrm{t} / \mathrm{min}$. Le rotor, en alliage d'aluminium, a $16 \mathrm{~cm}$ de diamètre et $1 \mathrm{~m}$ de long. Le bobinage formé de barres Roebell dans lesquelles les fils de base ont un diamètre de $1 \mathrm{~mm}$, a la structure hélicoïdale décrite figure 7 [2]. Nous avons, pour ce prototype, effectué le calcul des pertes par courants de Foucault et par courants de circulation apparaissant dans le bobinage ainsi que celles qui existeraient si le bobinage était réalisé avec des barres torsadées formées à partir de minces fils de base, le rayon $r_{t}$ étant de $1 \mathrm{~cm}$ pour avoir la même épaisseur de bobinage.
4.1.1 Pertes par courants de Foucault. - Nous avons tout d'abord considéré le cas où le stator est alimenté par un courant purement sinusoïdal de fréquence $\omega / 2 \pi$, ce qui provoque des pertes Joule «directes » $P_{\mathrm{J}} ;$ la pulsation rotorique choisie $(28 \mathrm{rad} / \mathrm{s})$ est celle qui donne le couple maximal du prototype. On trouve :

- pour les barres Roebell

$$
\frac{P_{\mathrm{F}}}{P_{\mathrm{J}}}=7,26 \times 10^{-8}(\omega / 2 \pi)^{2}
$$

- pour les barres torsadées

$$
\frac{P_{\mathrm{F}}}{P_{\mathrm{J}}}=7,50 \times 10^{-8}(\omega / 2 \pi)^{2} .
$$

On constate que les pertes Foucault dans les barres Roebell et les barres torsadées sont quasiment les mêmes. Elles restent toujours très faibles par rapport aux pertes Joule directes (qui représentent à peine $10 \%$ de la puissance active): $P_{\mathrm{F}} / P_{\mathrm{J}}$ vaut $1,8 \%$ à $500 \mathrm{~Hz}$, fréquence maximale admissible conduisant à la vitesse périphérique maximale autorisée pour le rotor.

Sur la figure 8 , nous avons porté $P_{\mathrm{F}} / P_{\mathrm{J}}$ en fonction de la pulsation rotorique $\omega_{R}$ (pour $\omega / 2 \pi=350 \mathrm{~Hz}$ et pour les barres torsadées). Nous constatons que les pertes Foucault sont toujours très faibles par rapport aux pertes Joule directes, ce qui rend quasiment impossible toute vérification expérimentale sur le prototype.

Nous avons ensuite calculé les pertes dans le cas d'une alimentation par un courant de forme trapézoïdale (cf. Fig. 9) très voisine de celle qui est fournie par un onduleur. Sachant que $[\mathrm{AB}]+[\mathrm{BC}]$ représente $60^{\circ}$ électriques, nous avons fait varier la «pente » $\mathrm{AB}$ de $20^{\circ}$ à $40^{\circ}$; cette dernière valeur transforme pratiquement les trapèzes en créneaux. Les résultats obtenus sont consignés dans le tableau I. Nous y avons indiqué :

- l'importance du premier harmonique non nul : il est à $5 \omega$ et représente environ $20 \%$ du fondamental ; 


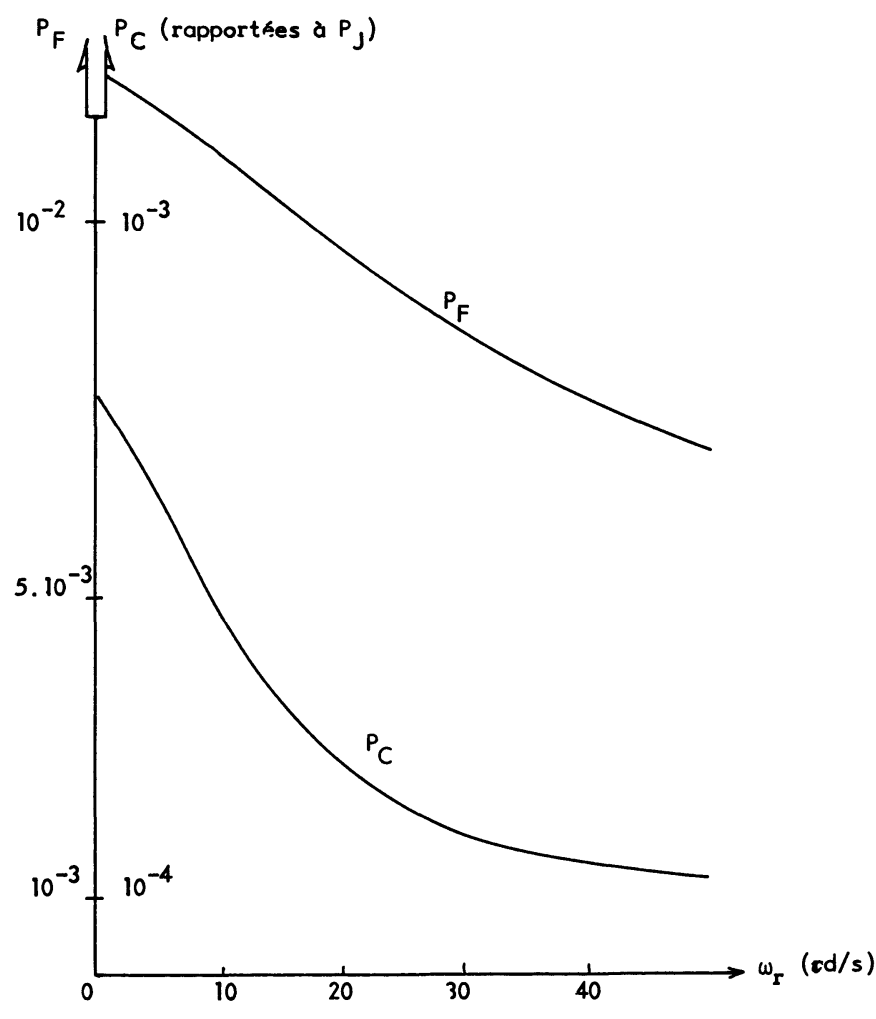

Fig. 8. - Pertes par courant de Foucault et par courants de circulation comparées aux pertes Joule en fonction de la pulsation rotorique.

[Eddy currents losses and circulating currents losses compared to Joule losses as a function of the rotoric pulsation.]

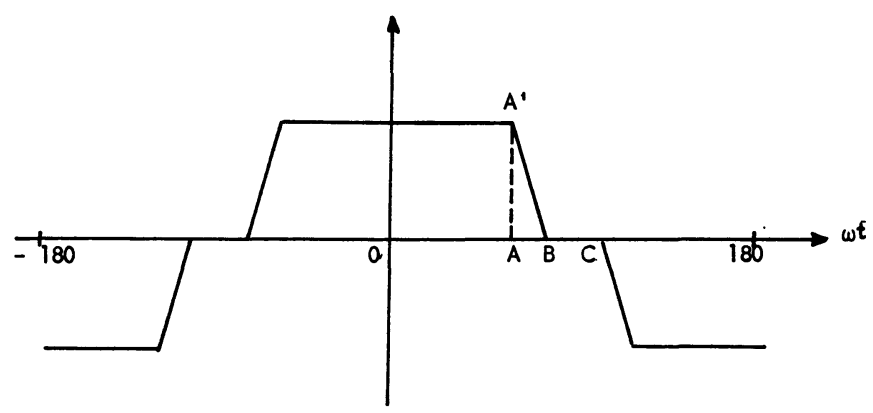

Fig. 9. - Courant de forme trapézoïdale.

[Current with a trapezoïdal form.]

- le rapport entre les pertes Joule directes et la puissance de la machine ;

- le rapport entre les pertes Foucault dues à l'harmonique $5 \omega$ et celles dues au fondamental;

- le rapport entre les pertes Foucault dues à l'ensemble des harmoniques et celles dues au fondamental.

Ce calcul a été effectué pour une fréquence d'alimentation de $350 \mathrm{~Hz}$ pour le glissement $\omega_{\mathrm{R}}=$ $28 \mathrm{rad} / \mathrm{s}$ conduisant au couple maximal, et pour des barres Roebell. En fait, les pertes par courants de
Tableau I. - Pertes Foucault dues à l'harmonique $5 \omega\left(P_{\mathrm{F}}(5 \omega)\right)$ et pertes Foucault totales $\left(P_{\mathrm{F}}\right)$ comparées à celles dues au fondamental $\left(P_{\mathrm{F}}(\omega)\right)$.

[Eddy losses at the frequency $5 \omega\left(P_{\mathrm{F}}(5 \omega)\right)$ and ratio between the total eddy losses $\left(P_{F}\right)$ and the eddy losses at the frequency $\omega\left(P_{F}(\omega)\right)$.]

\begin{tabular}{|c|c|c|c|c|}
\hline Pente (él.) & $20^{\circ}$ & $10^{\circ}$ & $8^{\circ}$ & $4^{\circ}$ \\
\hline$I_{5} / I_{1}$ & 0,176 & 0,194 & 0,196 & 0,199 \\
\hline$P_{\mathrm{J}} /$ Puis. & $10,6 \%$ & $10,7 \%$ & $10,8 \%$ & $10,9 \%$ \\
\hline$P_{\mathrm{F}}(5 \omega) / P_{\mathrm{F}}$ & 0,172 & 0,208 & 0,213 & 0,219 \\
\hline$P_{\mathrm{F}} / P_{\mathrm{F}}(\omega)$ & 1,18 & 1,23 & 1,24 & 1,25 \\
\hline
\end{tabular}

Foucault calculées pour un harmonique $n \omega$ bien précis peuvent se mettre sous la forme :

$$
P_{\mathrm{F}}(n \omega)=K_{n} n^{2} \omega^{2}\left|I_{n}\right|^{2}
$$

où $K_{n}$ est caractéristique de l'harmonique $n$, dépend de $\omega_{\mathrm{R}}$ et pratiquement pas de $\omega$. $K_{n}$ et $I_{n}$, en général, décroissent rapidement avec $n$ mais le facteur $n^{2}$, lui, augmente. Ici $K_{5} / K_{1}=0,134$ et $I_{5} / I_{1} \sim 0,2$; par suite $P_{\mathrm{F}}(5 \omega) / P_{\mathrm{F}}(\omega) \sim 0,2$, les harmoniques supplémentaires modifient peu $P_{\mathrm{F}}(w)$.

4.1.2 Pertes par courants de circulation. - Elles concernent le bobinage fabriqué avec les barres torsadées constituées de fils de diamètre $1 \mathrm{~mm}$ et pour lesquelles $r_{\mathrm{t}}=1 \mathrm{~cm}$.

La figure 10 montre comment varient ces pertes lorsque l'on modifie la longueur du pas $\ell_{\mathrm{t}}$ d'enroulement des fils. On voit qu'elles diminuent fortement avec $\ell_{t}$, c'est-à-dire lorsque les flux embrassés par les

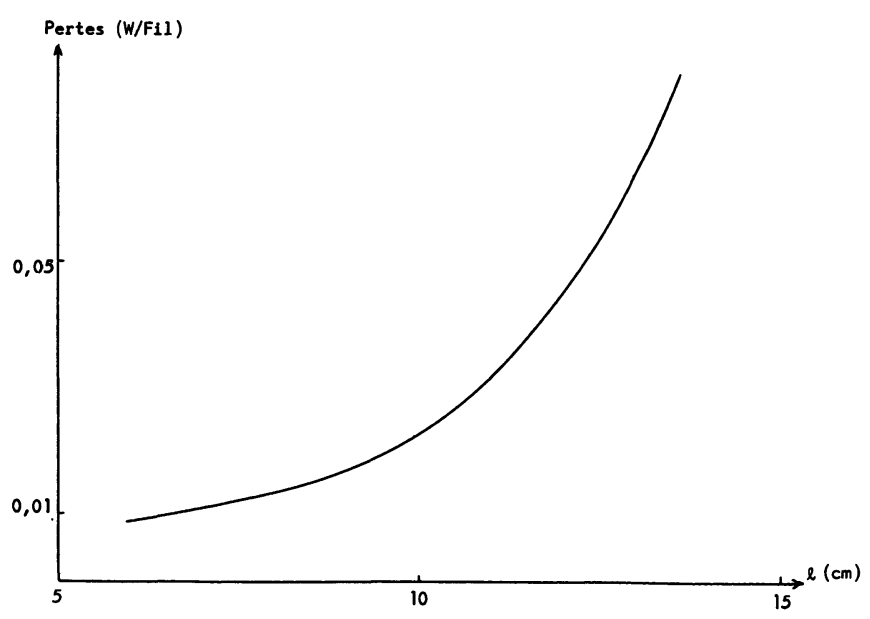

Fig. 10. - Pertes par courants de circulation en fonction du pas d'enroulement des fils dans le toron.

[Circulating currents losses as a function of the winding pitch.] 
différents pas d'un fil se compensent plus facilement : si l'on divise $\ell_{\mathrm{t}}$ par 2 , passant de 7 à 14 pas sur la longueur de la machine, les pertes sont divisées par 6.

Sur la figure 8 , où nous avions déjà porté les pertes par courants de Foucault $P_{\mathrm{F}}$ pour ce bobinage, nous avons aussi porté les pertes par courants de circulation $P_{\mathrm{c}}$ lorsque $\ell_{\mathrm{t}}=6,1 \mathrm{~cm}$ (soit 16,3 pas sur la longueur de la machine), ce qui correspond à un pas assez serré. On voit qu'elles sont faibles par rapport aux pertes par courants de Foucault : pour le glissement donnant le couple maximal, le rapport est de $2 \times 10^{-2}$ pour la valeur $\ell_{t}=6,1 \mathrm{~cm}$, il serait de 0,14 pour $\ell_{t}=14 \mathrm{~cm}$. Ce résultat incite à ne pas calculer les courants de circulation dans les barres Roebell (pour lesquelles la surface traversée par le flux est quasiment nulle) car ils seraient beaucoup plus petits et conduiraient à des pertes tout à fait négligeables.

4.2 Pertes DANS Les MAChines SANS Fer. - Les machines sans fer sont destinées à être réalisées sous grande dimension et à fournir des fortes puissances. En effet, leur puissance est proportionnelle à :

$$
P=k H^{2} v R^{2} .
$$

$H$ est l'ordre de grandeur du champ dans la machine : $H=k^{\prime} j R$ où $j$ est la densité de courant dans le stator. $k$ et $k^{\prime}$ sont des coefficients de forme géométrique (dépendant de $L / R, S_{\mathrm{i}} / R, S_{\mathrm{e}} / R$, cf. Fig. 5) ; $v$ est la vitesse périphérique du rotor, $v=\omega R$.

Les pertes Joule dans le stator valent :

$$
P_{\mathrm{J}}=j^{2} V_{\mathrm{s}} / \sigma=k_{\mathrm{J}} H^{2} R / \sigma
$$

$k_{\mathrm{J}}$ étant aussi un coefficient géométrique.

$$
\text { On a donc } \quad \frac{P_{\mathrm{J}}}{P}=\frac{k_{\mathrm{J}}}{k} \cdot \frac{1}{\sigma v R} .
$$

La vitesse périphérique maximale admissible pour le rotor est liée aux caractéristiques du matériau avec lequel il a été fabriqué. Les pertes Joule relatives décroissent donc comme $1 / R$ et seront ainsi de quelques pour cent pour les machines de grandes dimensions, fournissant quelques MW.

Les pertes par courants de Foucault sont, d'après (5) ou (7) telles que :

$$
P_{\mathrm{F}}=k_{\mathrm{F}} \sigma r_{\mathrm{c}}^{2} H^{2} \omega^{2} R^{3}=k_{\mathrm{F}} \sigma r_{\mathrm{c}}^{2} H^{2} v^{2} R
$$

$k_{\mathrm{F}}$ étant un coefficient lié à la géométrie de la machine.

Par suite :

$$
\frac{P_{\mathrm{F}}}{P_{\mathrm{J}}}=\frac{k_{\mathrm{F}}}{k_{\mathrm{J}}} \sigma^{2} r_{\mathrm{c}}^{2} v^{2}
$$

Lorsque l'on construit des machines de grandes dimensions, il est naturel de prendre des barres Roebell ayant des sections importantes qui sont fabriquées à partir de fils de rayon $r_{\mathrm{c}}$ grand. Les pertes Foucault étant liées à $H^{2}$, elles sont concentrées dans la partie centrale du bobinage (cf. Fig. 6) et peuvent alors dans cette région accroître de façon notable l'élévation de température due aux pertes Joule directes (le calcul que nous avons mis en place, peut, pour chaque configuration, préciser cette valeur si nécessaire). C'est pourquoi, on peut être amené à utiliser des barres torsadées qui peuvent être fabriquées à partir de fils fins, ce qui n'est pas possible pour les Roebell.

Les pertes par courants de circulation sont, d'après (14), telles que :

$$
P_{\mathrm{c}}=j_{b}^{2} V_{\mathrm{s}} / \sigma=k_{\mathrm{c}} \sigma r_{\mathrm{t}}^{2} H^{2} v^{2} R
$$

$k_{\mathrm{c}}$ étant un coefficient dépendant de la géométrie de la machine et de la longueur $\ell_{t}$ du pas des torons des barres.

On a donc :

$$
\frac{P_{\mathrm{c}}}{P_{\mathrm{F}}}=\frac{k_{\mathrm{c}}}{k_{\mathrm{F}}} \cdot \frac{r_{\mathrm{t}}^{2}}{r_{\mathrm{c}}^{2}}
$$

Ce rapport est très sensible aux variations des rayons $r_{\mathrm{t}}$ et $r_{\mathrm{c}}$ mais encore plus à celle de $\ell_{\mathrm{t}}$ (cf. Fig. 10). Les calculs effectués sur le prototype nous montrent, ce qui n'était pas évident a priori, qu'une géométrie « appropriée " des barres torsadées (grand nombre de pas sur la longueur de la machine, valeur de $r_{t}$ relativement peu importante) permet de rendre négligeables les courants de circulation. Cependant, un calcul précis est nécessaire pour bien définir la géométrie "appropriée » à chaque cas, ce que permet la méthode décrite ici.

\section{Conclusion.}

Nous avons mis en place une méthode qui permet, à partir de la modélisation des machines sans fer existante, de déterminer de façon rapide et précise, les courants de Foucault et de circulation apparaissant dans les conducteurs du stator. Nous avons ainsi constaté que nous pouvons toujours choisir des conducteurs pour lesquels ces deux types de courants conduisent à des pertes négligeables, par exemple en prenant des barres torsadées fabriquées à partir de fils très fins avec un petit pas d'enroulement. Mais ces dernières étant plus difficiles à fabriquer que les barres Roebell, il est bon de disposer d'une modélisation qui permettra un choix en fonction des performances requises. 


\section{Bibliographie}

[1] Rioux C., Bleiss C. A., Rioux-Damidau F., SultaNEM F., Machines sans fer : éléments de comparaison entre calculs et essais sur maquette, Rev. Gen. Elec. 10 (1984) 619.

[2] Rioux-Damidau F., Calcul semi-tridimensionnel des champs et des courants dans les machines asynchrones sans fer, Revue Phys. Appl. 18 (1983) 113.

[3] Belot A., Pertes dans les machines tournantes, Techniques de l'Ingénieur, D441-442, Paris (1982).

[4] Nithart J. J., Kiblaire M., Calcul des dimensions d'un câble transposé comprenant $n$ fils de cuivre émaillés. Note LTI $n^{\circ} 81067$, Alsthom-Atlantique, Belfort (1981).

[5] ISOLA, fabrique suisse d'isolants, torons en fils émaillés, laminés méplats. Feuille technique $n^{\circ}$ 24/009 (1972), The Swiss Insulating Works Ltd, CH-4226 Breitenbach.

[6] Bossavit A., Vérité J. C., A mixed FEM-BIEM method to solve 3D eddy-current problems, IEEE Trans. Magn. MAG-18 (1982) 431-435.
[7] EMSON C. R. I., Simkin J. and TROWbRidge C. W., Further Developments in Three Dimensional Eddy Current Analysis, IEEE Trans. Magn. MAG-21 (1985) 2231.

[8] KAMEARI A., Three Dimensional Eddy Current Calculation Using Finite Element Method with $\mathrm{A}-\mathrm{V}$ in Conductor and $\Omega$ in Vacuum, IEEE Trans. Magn. MAG-24 (1988) 118.

[9] GuÉraud A., Contribution à l'étude des machines électriques à conducteurs dans l'entrefer, Thèse de Doctorat, Université Paris VI (1988).

[10] BANDEliER B., Contribution à la modélisation des machines sans fer par séries de Fourier : étude d'un cas de couplage d'harmoniques et calcul des performances liées à la structure de divers bobinages, thèse de Doctorat, Université Paris VI (1986). 